\title{
Effect of interscalene brachial plexus block on heart rate variability
}

\author{
Marina Simeoforidou ${ }^{1}$, George Vretzakis ${ }^{1}$, Eleni Chantzi ${ }^{1}$, Metaxia Bareka ${ }^{1}$, Katerina Tsiaka ${ }^{1}$, \\ Christos Iatrou $^{2}$, and Theophilos Karachalios ${ }^{3}$ \\ ${ }^{1}$ Anesthesiology Clinic, University of Larissa, Larissa, Greece, ${ }^{2}$ Anesthesiology Clinic, Democritus University of Thrace, Komotini, Greece, \\ ${ }^{3}$ Orthopedic Clinic, University of Larissa, Larissa, Greece
}

Background: Interscalene brachial plexus block (ISB) may be followed by cardiovascular instability. Until date, there is no clear picture available about the underlying mechanisms of ISB. In this study, we aimed to determine the changes in heart rate variability (HRV) parameters after ISB and the differences between right- and left-sided ISBs.

Methods: We prospectively studied 24 patients operated for shoulder surgery in sitting position and divided them into two respective groups: $\mathrm{R}$ (right-sided block $=14 \mathrm{pts}$ ) and L (left-sided block $=10 \mathrm{pts}$ ). HRV data were taken before and 30 min after the block. Ropivacaine without ephedrine was used for the ISB through an insulated block needle connected to a nerve stimulator. Statistical analysis implemented chi-square, Student's and t-paired tests. Skewed distributions were analyzed after logarithmic transformation.

Results: All the studied patients had successful blocks. Horner's syndrome signs were observed in $33.3 \%$ of the patients $(\mathrm{R}=5 / 14, \mathrm{~L}=3 / 10 ;[\mathrm{P}=0.769])$. There were no significant differences in pre-block HRV between the groups. The application of ISB had differential effect on HRV variables: R-blocks increased QRS and QTc durations and InPNN50, while a statistical decrease was seen in InLF. L-blocks did not show any significant changes. These changes indicate a reduced sympathetic and an increased parasympathetic influence on the heart's autonomic flow after R-block.

Conclusions: Based on the obtained results we conclude that ISB, possibly through extension of block to the ipsilateral stellate ganglion, alters the autonomic outflow to the central circulatory system in a way depending on the block's side. (Korean J Anesthesiol 2013; 64: 432-438)

Key Words: Bradycardia, Heart rate variability, Hypotension, Interscalene block, Stellate ganglion block.

Received: September 6, 2012. Revised: 1st, October 12, 2012; 2nd, October 24, 2012. Accepted: October 29, 2012.

Corresponding author: George Vretzakis, M.D., Ph.D., Anesthesiology Clinic, University of Larissa, Mezourlo, Larissa 41110, Greece. Tel: 00302413502952, Fax: 00302413501017, E-mail: gvretzakis@yahoo.com

(c) This is an open-access article distributed under the terms of the Creative Commons Attribution Non-Commercial License (http:// creativecommons.org/licenses/by-nc/3.0/), which permits unrestricted non-commercial use, distribution, and reproduction in any medium, provided the original work is properly cited. 


\section{Introduction}

Interscalene brachial plexus block (ISB) is often selected by skilled anesthesiologists as the primary anesthetic technique for shoulder surgery. A disadvantage of the technique is the cardiovascular instability, such as bradycardia and/or hypotension that may be seen in up to $29 \%$ of the patients operated in sitting position [1-7]. The underlying mechanisms of ISB are still not well understood. For the interpretation of this side effect, a cardioinhibitory mechanism by receptors mediating the Bezold-Jarisch reflex, similar to a form of vasovagal syncope, has been proposed $[2,8]$. Conflicting data exist about the impact of the use of epinephrine as an additive to the local anesthetic solution $[5,7]$. As stellate ganglion block is frequently observed along with ISB [6], an association of this block with bradycardia and/or hypotension has been proposed [9]. Furthermore, this extension of the block, from brachial plexus to stellate ganglion, may explain the different incidence of cardiovascular instability between right and left-sided ISBs $[2,7]$. The autonomic cardiovascular control exhibits a sympathetic predominance in the right side and a parasympathetic predominance in the left side.

Heart rate variability (HRV) represents a simple and noninvasive electrocardiographic quantitative index of the heart's autonomic status. HRV shows a component of self-similarity (fractal) dynamics. Loss of complexity of HRV equals with increased fractal component of heart rate fluctuations, and has been used to predict cardiac mortality and morbidity. In reports implementing HRV, right or left-sided stellate ganglion blocks has been demonstrated to have different impact on the autonomic status of the heart $[10,11]$. Although many investigators suspect the association of cardiovascular instability during ISB to a complicating stellate ganglion block, until date there is no available data on autonomic outflow during ISB. In this investigation, the cardiac autonomic status of patients who underwent shoulder surgery in sitting position under ISB was studied along with HRV analysis. The main purpose of our study was to elucidate whether or not ISB differentiates complexity of fractal heart rate. In this prospective trial, we are testing the a priori hypotheses that there is no difference in HRV parameters both a) before and after ISB, and $b$ ) between patients receiving right or left-sided ISB.

\section{Materials and Methods}

This prospective study was conducted in a tertiary care University Hospital over a 2-year period, after obtaining approval from the Institution Ethics committee. All patients gave their written informed consent before entering the study. The study was registered at the www.clinicaltrials.gov registration site, (registration number NCT01185457). Interscalene brachial plexus blocks were performed by two anesthesiologists (CE, $\mathrm{SM}$ ) with extensive experience in this technique. Both they preferred this block for shoulder surgery in sitting position. Since June 2010 and for a period of two years, all adult patients scheduled for elective arthroscopic or open shoulder surgery who were assigned by our clinic's program to the abovementioned specialists, were considered eligible for inclusion in our study. Surgeries of upper arm or surgeries done using a tourniquet were not included. Inclusion was based on the patient's willingness and ability to comprehend and collaborate. Exclusion criteria were body mass index $>35$, coagulation deficiencies, known allergies to local anesthetics, neurologic disorder in the limb, which was to be operated, inflammation in the region, coronary artery disease, arrhythmia, conduction disorders in the electrocardiogram, congestive heart failure, diabetes mellitus, abnormal thyroid hormones, renal insufficiency, abnormal serum electrolytes and autonomic dysfunction. The preoperative anesthetic evaluation and the establishment of a peripheral venous access were done in the afternoon before surgery by the responsible anesthesiologist along with the one who was assigned to take the HRV values. Patients were not premedicated, and anesthetic and surgical procedures were performed by the same team under standardized conditions.

In the morning of the day of surgery, patients were taken to a separate room in our Post-anesthesia Care Unit for HRV measurements and for the application of ISB. They were connected to a "silent" monitor for 5-lead electrocardioscopy, pulse oximetry and on demand non-invasive measurement of arterial blood pressure. Monitor-displayed baseline values were recorded. Baseline (pre-block, sample 1) and post-block (sample 2) HRV values were taken by the same anesthesiologist (BM, TK) in this quiet, comfortable environment with dim lighting and a temperature between $23^{\circ}$ and $24^{\circ} \mathrm{C}$. Sample 1 was recorded before any painful stimuli, with the patient calm and in supine position. An effort was made to provide similar conditions for obtaining sample 2. In all the subjects, we used the Schiller Cardiovit AT-60 electrocardiograph (Schiller AG, Baar, Switzerland) and the built-in software. Heart rate, duration of $\mathrm{RR}, \mathrm{P}, \mathrm{PR}, \mathrm{QRS}, \mathrm{QT}$ and QTc intervals were entered in a database from the Holter instrument's printed report. Cardiac autonomic function was assessed by time and frequency-domain HRV analysis on 512 consecutive normal RR intervals, corresponding to a time window of a few minutes. Indices in the time domain analysis were taken in the nocorrecture mode and included the average duration of normal RR (normal sinus to normal sinus) intervals (average NN, in milliseconds), the standard deviation of RR intervals (SDNN, in $\mathrm{ms}$ ), the percentage of successive RR intervals greater than 
50 milliseconds (tachogram PNN50, in percentages), and the square root of the averaged sum of squared differences in length between all adjusted RR cycles (RMSSD, in percentages). Frequency-domain HRV was assessed in the range of 0 to $1 \mathrm{~Hz}$ by fast-Fourier transform spectral analysis. To improve signal processing, a low-resolution (high-dynamic range) window function (Blackman-Harris) was selected. According to the selected fast-Fourier transform mode, frequency variables were presented as dimensionless numbers and as percentage of the total power. The following frequency domain HRV variables were obtained: low frequency (LF), 0.04 to $0.15 \mathrm{~Hz}$; high frequency (HF), 0.15 to $0.40 \mathrm{~Hz}$; and the ratio of low to high frequency (LF/HF). Generally, HF primarily reflects parasympathetic nervous system activity and LF reflects both parasympathetic and sympathetic nervous system activity. LF is influenced more by sympathetic activity. The ratio of the low frequency power spectrum components to the high frequency components (LF/ $\mathrm{HF}$ ) represents the balance between the two systems. When it decreases, a deflection to parasympathetic dominance is suggested.

After taking HRV-sample 1 measurement, a bolus dose of $0.30-0.40 \mathrm{mg}$ of alfentanil was given and the single-injection ISBs were performed in supine position with patient's head turned to opposite side. The interscalene groove was identified and the area was sterilized and anesthetized with a subcutaneous injection of $1-2 \mathrm{ml}$ of $1 \%$ lidocaine, using a 25-gauge needle. After waiting for loss of sensation in the area, the ISB was performed by using a sterile 22-gauge 50 $\mathrm{mm}$ insulated block needle connected to a nerve stimulator (Stimuplex; B.Braun, Melsungen, Germany). The initial current output of the stimulator was set at $1.2 \mathrm{~mA}$. The needle was advanced through the interscalene groove until contractions of biceps or/and forearm musculature were provoked. The position of the needle was subsequently adjusted so that contractions were still present with a current output of 0.5 $\mathrm{mA}$. In this position, $40 \mathrm{ml}$ of a pre-prepared solution of $0.5 \%$ ropivacaine without epinephrine was injected after connecting the needle-extension tubing assembly via a three-way stopcock to a local anesthetic syringe. Thirty minutes after the injection of local anesthetic, the degree of the sensory blockade was tested by loss of pinprick sensation in the C5-6 dermatomal distribution and the motor blockade by ordering the patient to raise the straightened arm against gravity. Blocks were graded as: no block $=0$, moderate block $=1$, and complete block $=2$. In case of adequate anesthesia (grade 2), the patient was reconnected to the Holter instrument for the HRV-sample 2 measurement which, by our protocol, was taken 30 minutes after the initial ISB. After taking HRV values, the patient was transported to the operating room for the surgical procedure. All surgeries were performed with the patient in the sitting position, commonly known as "beach chair" position (elevation of the back of the table and flexion of patient's knees and hips). In case of arthroscopic surgery, the irrigating fluid did not contain epinephrine. For these surgeries, an intravenous dose of 75 micrograms of clonidine was administered if patient's blood pressure impaired visualization. Patients showing block grade $<2$ or with a requirement of intravenous sedatives or analgesics were excluded from the analysis.

Power analysis for sample size estimation was done with the natural logarithm of LF (InLF) as primary outcome variable. Because relevant literature to our a priori hypotheses was limited, we based the present calculation on data from a previous study conducted by us where HRV was measured in a similar manner [12], and on the assumption that a change of $\pm 20 \%$ is clinically meaningful. Henceforth, the study required 9 patients for one group when alpha was set at 0.05 and power at $\mathbf{8 0 \%}$. Considering the per year number of elective shoulder operations in our Hospital, we registered the study with June 2012 as completion date thus allowing us to enroll more patients for attrition and missing data.

Data concerning patient's demographic characteristics and physical status, type, site and duration of operation, duration of anesthesia, fluid administration, intraoperative use of medications, and episodes of bradycardia or hypotension were recorded on a standardized data collection sheet. Observation period lasted for 2 hours after termination of operation. Bradycardia was defined as $\mathrm{HR}<50 \mathrm{bpm}$ and hypotension was defined as mean arterial pressure (MAP) $<60 \mathrm{mmHg}$ or MAP $<70 \mathrm{mmHg}$ together with a decrease of $>30 \%$ as compared to baseline value. Data concerning Horner's syndrome signs (ptosis of the upper eyelid, miosis and dilation lag, enophthalmos) were also recorded. These data and data from HRV measurements were stored electronically in Excel and were analyzed with SPSS 15.0 for Windows (SPSS Inc, Chicago, IL, USA). Data were expressed as mean \pm standard deviation. Demographic and clinical patient characteristics were compared between groups using the chi-square test for categorical data and the Student's 2-tailed t-test and t-paired test for continuous data. Continuous data normality was tested with the Kolmogorov-Smirnov test. Correction of skewed distributions was performed after adequate normalization using natural logarithmic transformation and our data are presented in this form. A P value of less than 0.05 was considered significant.

\section{Results}

In the entire 2-year study period, 286 patients were referred to our Anesthesiology Clinic for elective shoulder surgery. From them, 46 were assigned to the study team. After excluding 
the subjects due to participation refusal or unwillingness for regional anesthesia or due to protocol criteria, pre- and postblock HRV measurements (samples 1 \& 2) were taken from 26 of them. Two patients complained for pain early in the beginning of the surgical procedure, although initially their block was graded as 2 (complete block). Consequently, their anesthetic support was changed and they were excluded from the analysis. Finally, 24 patients were studied, divided into two groups according to the operation site, namely group $\mathrm{R}$ (right-sided ISB, 14 patients) and group L (left-sided ISB, 10 patients). They were operated in sitting position with anesthesia provided by ISB, which was graded as 2 , with no feeling of pain and without any intravenous analgesic support. No case of missing data was noted. Baseline demographic and clinical characteristics did not differ significantly between the groups (age [yr]: $\mathrm{R}=38.2 \pm$ 8.4, $\mathrm{L}=32.1 \pm 11.4[\mathrm{P}=0.132]$; body weight $[\mathrm{kg}]: \mathrm{R}=68.6 \pm 14.1, \mathrm{~L}$ $=72.2 \pm 11.6[\mathrm{P}=0.097]$; height $[\mathrm{cm}]: \mathrm{R}=169.0 \pm 10.0, \mathrm{~L}=175.2 \pm$ $11.2[\mathrm{P}=0.288]$; gender: $\mathrm{R}:$ male $=11[78.6 \%]$, female $=3[21.4 \%]$, $\mathrm{L}:$ male $=9[90 \%]$, female $=1[10 \%],[\mathrm{P}=0.458]$; American Society of Anesthesiologists physical status classification: $\mathrm{R}: \mathrm{I}=$ 10 [71.4\%], II = 4 [28.6\%], L: I = 7 [70\%], II = 3 (30\%), [P = 0.939]). Open surgery was performed in 5 patients belonging to group $\mathrm{R}$ (35.7\%) and in 3 belonging to group L (30.0\%) ( $\mathrm{P}=0.769)$, and the rest was arthroscopic. The mean time of observation period was $235.5 \pm 92.6 \mathrm{~min}$, which was not different between the groups.

There were no significant differences between groups in baseline heart rate values taken from the Holter instrument $(\mathrm{R}$
$=69.9 \pm 10.7 \mathrm{bpm}, \mathrm{L}=75.8 \pm 11.1 \mathrm{bpm} ; \mathrm{P}=0.232)$. Differences in duration of electrocardiographic intervals and time- and frequency domain indices of HRV were also insignificant between patients belonging to groups $\mathrm{R}$ and L (Table 1, sample 1). These parameters were differently affected among groups by the application of ISB. Right-sided ISB increased QRS and QTc durations (from $88.5 \pm 11.6 \mathrm{~ms}$ to $91.4 \pm 10.9 \mathrm{~ms}[\mathrm{P}=0.001]$ and from $401.2 \pm 20.4 \mathrm{~ms}$ to $419.2 \pm 22.2 \mathrm{~ms}$ [P $=0.005$ ], respectively) without significantly affecting HR (from $69.9 \pm 10.7 \mathrm{bpm}$ to $70.5 \pm 9.9 \mathrm{bpm})$. A statistical decrease in InPNN50 and InLF was observed in this group. Left-sided ISB affected only QRS duration while parameters of time- and frequency domain analyses of HRV did not exhibit any significant changes. After comparing the R \& L values after the application of ISB in both the groups, the only observed difference was the duration of $\mathrm{P}$ interval (121.4 $\pm 22.4 \mathrm{~ms}$ for $\mathrm{R}$ and $98 \pm 10.9 \mathrm{~ms}$ for $\mathrm{L}[\mathrm{P}=0.009]$ ). Two patients, distributed equally in groups $\mathrm{R}$ and $\mathrm{L}$, showed original values of PNN50 (\%) equal to zero in sample 1 or 2 or both. As this value could not be transformed in a natural logarithm, these 2 patients were excluded from InPNN50 analysis. Finally, Horner's syndrome signs were observed in 5 patients belonging to group R (35.7\%) and in 3 belonging to group L (30.0\%) $(\mathrm{P}=0.769)$. In all these patients, the signs were clearly noticeable 30 minutes after the block and lasted for at least an hour. None of the patients exhibited these signs at the time of departing from Post-anesthesia Care Unit.

There were no significant differences in baseline heart rate values (taken from the monitor before HRV-sample 1)

Table 1. Effects of Interscalene Brachial Plexus Block on ORS Intervals Duration of ECG, and Time- and Frequency-domain Variables of HRV

\begin{tabular}{|c|c|c|c|c|c|c|c|c|}
\hline & \multicolumn{3}{|c|}{ Sample 1} & \multicolumn{5}{|c|}{ Sample 2} \\
\hline & Right & Left & $\begin{array}{c}\text { P values } \\
\text { R, } L\end{array}$ & Right & $\begin{array}{c}\text { P values } \\
\text { samples } 1,2\end{array}$ & Left & $\begin{array}{c}\text { P values } \\
\text { samples 1, } 2\end{array}$ & $\begin{array}{c}\text { P values } \\
\text { R, } L\end{array}$ \\
\hline HR & $4.23 \pm 0.15$ & $4.31 \pm 0.14$ & 0.232 & $4.24 \pm 0.14$ & 0.719 & $4.28 \pm 0.18$ & 0.313 & 0.646 \\
\hline $\mathrm{RR}$ & $6.75 \pm 0.14$ & $6.67 \pm 0.15$ & 0.234 & $6.69 \pm 0.12$ & 0.111 & $6.67 \pm 0.21$ & 0.975 & 0.763 \\
\hline $\mathrm{P}$ & $4.70 \pm 0.11$ & $4.67 \pm 0.14$ & 0.606 & $4.78 \pm 0.19$ & 0.117 & $4.58 \pm 0.11$ & 0.171 & 0.013 \\
\hline PR & $5.04 \pm 0.09$ & $4.97 \pm 0.12$ & 0.135 & $5.07 \pm 0.17$ & 0.433 & $4.98 \pm 0.11$ & 0.769 & 0.196 \\
\hline QRS & $4.47 \pm 0.12$ & $4.52 \pm 0.14$ & 0.428 & $4.50 \pm 0.11$ & 0.002 & $4.54 \pm 0.12$ & 0.011 & 0.460 \\
\hline QT & $5.91 \pm 0.08$ & $5.91 \pm 0.07$ & 0.867 & $5.92 \pm 0.09$ & 0.255 & $5.91 \pm 0.08$ & 0.934 & 0.824 \\
\hline QTc & $5.99 \pm 0.05$ & $6.04 \pm 0.07$ & 0.122 & $6.03 \pm 0.05$ & 0.004 & $6.04 \pm 0.05$ & 0.970 & 0.876 \\
\hline Average NN & $6.76 \pm 0.15$ & $6.68 \pm 0.14$ & 0.231 & $6.74 \pm 0.13$ & 0.512 & $6.72 \pm 0.18$ & 0.290 & 0.750 \\
\hline SDNN & $3.76 \pm 0.36$ & $3.71 \pm 0.38$ & 0.735 & $3.66 \pm 0.35$ & 0.331 & $3.72 \pm 0.33$ & 0.916 & 0.682 \\
\hline PNN50 & $2.15 \pm 0.82$ & $1.23 \pm 1.12$ & 0.056 & $0.86 \pm 1.29$ & 0.004 & $1.33 \pm 1.07$ & 0.763 & 0.420 \\
\hline RMSSD & $1.01 \pm 0.40$ & $0.97 \pm 0.35$ & 0.775 & $0.55 \pm 0.60$ & 0.072 & $0.94 \pm 0.30$ & 0.749 & 0.080 \\
\hline LF & $5.65 \pm 0.78$ & $5.56 \pm 0.82$ & 0.785 & $4.90 \pm 0.90$ & 0.019 & $5.35 \pm 0.80$ & 0.422 & 0.251 \\
\hline $\mathrm{HF}$ & $4.95 \pm 0.87$ & $4.75 \pm 0.66$ & 0.570 & $4.13 \pm 1.27$ & 0.061 & $4.72 \pm 1.00$ & 0.883 & 0.259 \\
\hline LF/HF & $0.70 \pm 0.79$ & $0.80 \pm 0.67$ & 0.755 & $0.77 \pm 0.99$ & 0.763 & $0.62 \pm 0.74$ & 0.580 & 0.699 \\
\hline
\end{tabular}

Displayed values are logarithmic transforms; statistically significant if $\mathrm{P}<0.05$. Original values for HR (heart rate) in bpm, original values for $\mathrm{P}$, PR, QRS, QT, QTc in ms, original values for average NN (average duration of normal RR [normal sinus to normal sinus]) in ms, original values for SDNN (standard deviation of RR intervals) in ms, original values for PNN50 (the percentage of successive RR intervals greater than 50 ms) in percentages, original values for RMSSD (square root of the averaged sum of squared differences in length between all adjusted RR cycles) in percentages, LF \& HF are the logarithmic transforms of low and high frequency components of the power spectra, $\mathrm{LF} / \mathrm{HF}$ is the logarithmic transform of the ratio. 
Table 2. Changes in Hemodynamic Variables

\begin{tabular}{|c|c|c|c|c|c|c|}
\hline & \multicolumn{3}{|c|}{ Group R } & \multicolumn{3}{|c|}{ Group L } \\
\hline & Baseline value & Lowest value & $P$ value & Baseline value & Lowest value & $P$ value \\
\hline HR & $71.7 \pm 11.5$ & $60.7 \pm 9.4$ & 0.003 & $76.5 \pm 9.82$ & $59.4 \pm 8.5$ & 0.003 \\
\hline Sys AP & $133.1 \pm 19.4$ & $110.0 \pm 23.0$ & 0.003 & $124.6 \pm 18.1$ & $115.8 \pm 26.5$ & 0.377 \\
\hline Dia AP & $70.3 \pm 12.0$ & $57.0 \pm 13.4$ & 0.008 & $68.8 \pm 9.6$ & $64.8 \pm 21.3$ & 0.602 \\
\hline Mean AP & $91.4 \pm 12.7$ & $74.4 \pm 15.5$ & 0.002 & $87.5 \pm 11.6$ & $81.8 \pm 22.6$ & 0.498 \\
\hline
\end{tabular}

All values are the mean \pm standard deviation. Group R: right-sided ISB, 14 patients, Group L: left-sided ISB, 10 patients. HR (heart rate) in bpm; AP (arterial pressure) in mmHg. Baseline values = before the pre-block HRV measurement (sample 1), Lowest values = during the procedure, including HRV sampling, operation and postanesthesia care distributions of sys and dia AP were generated by values in respect to the lowest mean AP recording.

between patients belonging to groups $\mathrm{R}$ and $\mathrm{L}$ (Table 2). In both the groups, comparison of baseline values to the lowest values recorded during observation was statistically significant. Nevertheless, bradycardia (HR $<50 \mathrm{bpm}$ ) was not observed. Table 2 also displays the lowest mean arterial pressure during observation and the accompanying values of systolic and diastolic arterial pressures. No significant differences in baseline values between groups $\mathrm{R}$ and $\mathrm{L}$ was noticed. It can be seen in the table that arterial pressure dropped significantly at some instance of the procedure in group $\mathrm{R}$, while no change was observed in group L. In either of the groups, hypotension, as defined in our protocol, was also not observed. None of the patients received clonidine during the course of surgical procedure.

\section{Discussion}

Our study demonstrates that ISB has a differential effect on the autonomic outflow to the heart depending on the block's side. Right-sided blocks led to a decrease in the LF component of HRV suggesting a reduced sympathetic influence after the block, while left-sided blocks had no such effect. Moreover, in our patients, the activity of parasympathetic nervous system increased also after right-sided ISB, reflected in the timedomain analysis as statistical increase in PNN50 (proportion derived by dividing the number of successive RR intervals greater than $50 \mathrm{~ms}$ by the total number of normal-to-normal intervals), and in frequency-domain analysis as increased HF component not reaching statistical significance. Leftsided blocks left practically unaffected both the LF and HF components of HRV. Although statistically unimportant, a trend towards parasympathetic predominance after the application of left ISB was noted as decreased LF/HF ratio. Our results were noted in blocks without epinephrine in the local anesthetic solution and in patients who developed Horner's syndrome signs in a percentage of $33.3 \%$. In respect to our hypotheses, we suggest that ISB, possibly through extension of block to the ipsilateral stellate ganglion, alters the autonomic outflow to the central circulatory system and this influence depends on the block site.

Episodes of cardiovascular instability such as bradycardia and/or hypotension (BH) during shoulder surgery under interscalene block anesthesia have been reported by many investigators [1-7]. Mostly, these appear to be transient, isolated and uncomplicated, but there are a few cases where severe forms of BHs have occurred, including asystolic cardiac arrest [13-15]. The underlying mechanisms are not well understood and many possible causes of the observed symptomatology have been proposed. Existing data do not support causality from intravenous uptake of local anesthetic or from extensive spread to the epidural or subarachnoid space. A team of investigators suggested that the occurrence of cardiovascular instability during ISB is a form of vasovagal syncope and the underlying mechanism is the activation of cardioinhibitory mechanoreceptors or the Bezold-Jarisch reflex [2]. They observed that the total dose of epinephrine in the ISB was greater in patients experiencing $\mathrm{BH}$ while the patients who received systemic $\beta$-antagonist treatment were protected from these episodes. It has been postulated that endogenous as well as exogenous epinephrine, inducing a $\beta$-2 adrenergic effect, play a role similar to tilt-table testing in patients with unexplained syncope $[9,16]$. Further studies supported these hypotheses. In a prospective randomized study, the patients who received exogenous epinephrine showed significantly higher incidence of $\mathrm{BH}$ as compared with those who did not [5]. A prospective study reported that treatment with $\beta$-antagonist (metoprolol) was accompanied with significantly less episodes of BH during ISB for shoulder arthroscopy in sitting position [3]. However, these hypotheses were not confirmed by other investigators $[4,7]$. In fact, the available studies show a lack of consistency and in particular for the role of epinephrine it is difficult to conclude causality. Nevertheless, epinephrine can reduce the amount of local anesthetic which is absorbed systemically, leaving this way a greater proportion to diffuse by time to near-by anatomic formations such as stellate ganglia. We have to note that the use of epinephrine with this block is not practiced at all in our Clinic. We prefer other methods, like 
single-injection for ISB prolongation, when the duration of the procedure is expected long.

A limited number of studies report the operation site and thus the differences concerning $\mathrm{BH}$ between right and left cannot be easily identified. In a retrospective study of 116 patients with an incidence of $\mathrm{BH}$ of $17.2 \%$, the block site was $\mathrm{R} / \mathrm{L}=14 / 6$ for the patients showing $\mathrm{BH}$ vs $\mathrm{R} / \mathrm{L}=55 / 41$ for the patients not presenting $\mathrm{BH}$ [2]. In other words, the incidence of $\mathrm{BH}$ for right-sided blocks was $20.3 \%$ as compared to $12.7 \%$ for left-sided blocks. We propose that the authors wrongly stated that there was no predominance of right-side blocks in the $\mathrm{BH}$ group. More recently, in 63 patients studied retrospectively with a $20.6 \%$ incidence of $\mathrm{BH}$, the right operation side clearly favored the appearance of $\mathrm{BH}(\mathrm{R} / \mathrm{L}=12 / 1$ for the $\mathrm{BH}$ group vs $\mathrm{R} / \mathrm{L}=$ $32 / 18$ for the non-BH group) [7]. Recently, this relationship between $\mathrm{BH}$ and block side, with a contributing "intermediate" role of the ipsilateral stellate ganglion, is noted in a review addressing hypotensive and bradycardic events during ISB [9] and in an abstract of a prospective study implicating HRV analysis in ISB, where a significant percentage of patients showed Horner's syndrome signs [17]. Both, the review and the abstract were published after our decision to proceed with this study.

Interscalene brachial plexus block may be complicated with stellate ganglion block and induce Horner's syndrome [6,18-21]. Spreading of the local anesthetic solution injected, similar to the one observed during other blocks, cannot be excluded [22]. Information about the true incidence of Horner's syndrome during ISB, its relationship with the kind and dose of local anesthetic solution and the use of epinephrine as additive to this solution, or the block technique are scarce. In the past, it has been reported that in 248 patients with ISB, 46 (18.5\%) developed Horner's syndrome [23]. More recently, the reported incidence reached to $53.3 \%$ [17]. Nevertheless, in prospective studies dealing with the complications of ISB in shoulder and upper arm surgery, the induction of Horner's syndrome is not mentioned [24]. These studies, however, were not focused on anesthetic complications and thus circulatory instability, respiratory dysfunction or others are not mentioned as well.

Stellate ganglia provide sympathetic fibers to the heart and blockage of them might affect cardiac function thus inducing clinical symptomatology with many similarities to the cardiovascular instability seen with ISBs. The hemi-lateralization of the autonomic cardiovascular control results in different effects between right and left stellate ganglion block. The autonomic cardiovascular control shows a sympathetic predominance in the right side and a parasympathetic predominance in the left. Infiltration of right stellate ganglion with a local anesthetic decreases heart rate while of left does not have any effect $[25,26]$. As with ISB, sinus arrest followed by apnea and unconsciousness has been reported with right SGB [27]. Presently, information about the impact of stellate ganglion block (SGB) on HRV is somehow inconsistent. In a study conducted using controlled head-up tilt test during SGB, it was found that both right and left SGB decreased baroreflex sensitivity and caused an imbalance in autonomic nerve activity with decreased cardiac sympathetic outflow manifested as a reduction in LF/HF ratio [10]. The authors demonstrated that right SGB affected both heart rate and systolic blood pressure variability, but left SGB affected only systolic blood pressure and had no effect on heart rate variability. They also noticed that all the studied subjects showed Horner signs in the ipsilateral side. Another study implicating HRV analysis and head-up tilt test during only right SGB found that tilting significantly increased mean $\mathrm{HR}$ and LF/HF, whereas HF markedly decreased at the same. After the right SGB, tilting had no effect on these parameters. The authors concluded that right SGB suppresses cardiac sympathetic function [28]. Another study found that right SGB did not result in any changes in sympathetic and parasympathetic activity, while left SGB increased parasympathetic activity [11]. In another study, both the LF and HF components of HRV were significantly decreased in right SGB while left SGB changed neither of them. Total frequency component was also decreased significantly during right SGB but was unchanged in left SGB [29]. These findings support predominance of the right side in the autonomic innervation of the sinus node.

The main finding of our study is that the peripheral (plexus) interscalene blockade can affect heart's autonomic status and secondly that this happens in a manner depending on the block side. Strengths of our study include inclusion of a well-defined patient population, prospective character, and avoidance of use of epinephrine with the local anesthetic. It is important to emphasize that our findings are in accordance and, at the same time, provide an explanation for observations such as the different incidence of $\mathrm{BH}$ between right- and left sided blocks. Disadvantage of the study is the limited number of studied patients, which does not allow us to clear out any differences in HRV values between patients developing Horner signs and those who do not, and between patients exhibiting $\mathrm{BH}$ and those who do not. However, we believe that, despite this important limitation, our findings are still worth reporting, because they suggest that, even under optimal conditions, expansion of ISB to other, closely sited, anatomical formations is not rare and plays a significant role in the development of circulatory instability.

\section{References}

1. D'Alessio JG, Rosenblum M, Shea KP, Freitas DG. A retrospective comparison of interscalene block and general anesthesia for 
ambulatory surgery shoulder arthroscopy. Reg Anesth 1995; 20: 62-8.

2. D'Alessio JG, Weller RS, Rosenblum M. Activation of the BezoldJarisch reflex in the sitting position for shoulder arthroscopy using interscalene block. Anesth Analg 1995; 80: 1158-62.

3. Liguori GA, Kahn RL, Gordon J, Gordon MA, Urban MK. The use of metoprolol and glycopyrrolate to prevent hypotensive/bradycardic events during shoulder arthroscopy in the sitting position under interscalene block. Anesth Analg 1998; 87: 1320-5.

4. Kahn RL, Hargett MJ. Beta-adrenergic blockers and vasovagal episodes during shoulder surgery in the sitting position under interscalene block. Anesth Analg 1999; 88: 378-81.

5. Sia S, Sarro F, Lepri A, Bartoli M. The effect of exogenous epinephrine on the incidence of hypotensive/bradycardic events during shoulder surgery in the sitting position during interscalene block. Anesth Analg 2003; 97: 583-8.

6. Brull R, McCartney CJ, Sawyer RJ, von Schroeder HP. The indications and applications of interscalene brachial plexus block for surgery about the shoulder. Acute Pain 2004; 6: 57-77.

7. Seo KC, Park JS, Roh WS. Factors contributing to episodes of bradycardia hypotension during shoulder arthroscopic surgery in the sitting position after interscalene block. Korean J Anesthesiol 2010; 58: $38-44$.

8. Campagna JA, Carter C. Clinical relevance of the Bezold-Jarisch reflex. Anesthesiology 2003; 98: 1250-60.

9. Song SY, Roh WS. Hypotensive bradycardic events during shoulder arthroscopic surgery under interscalene brachial plexus blocks. Korean J Anesthesiol 2012; 62: 209-19.

10. Taneyama C, Goto H. Fractal cardiovascular dynamics and baroreflex sensitivity after stellate ganglion block. Anesth Analg 2009; 109: 1335-40.

11. Kim JJ, Chung RK, Lee HS, Han JI. The changes of heart rate variability after unilateral stellate ganglion block. Korean J Anesthesiol 2010; 58: 56-60.

12. Simeoforidou M, Vretzakis G, Bareka M, Chantzi E, Flossos A, Giannoukas A, et al. Thoracic epidural analgesia with levobupivacaine for 6 postoperative days attenuates sympathetic activation after thoracic surgery. J Cardiothorac Vasc Anesth 2011; 25: 817-23.

13. Edde RR, Deutsch S. Cardiac arrest after interscalene brachialplexus block. Anesth Analg 1977; 56: 446-7.

14. Reinikainen M, Hedman A, Pelkonen O, Ruokonen E. Cardiac arrest after interscalene brachial plexus block with ropivacaine and lidocaine. Acta Anaesthesiol Scand 2003; 47: 904-6.

15. Turker G, Demirag B, Ozturk C, Uckunkaya N. Cardiac arrest after interscalene brachial plexus block in the sitting position for shoulder arthroscopy: a case report. Acta Orthop Belg 2004; 70: 84-6.

16. Kinsella SM, Tuckey JP. Perioperative bradycardia and asystole: relationship to vasovagal syncope and the Bezold-Jarisch reflex. Br J Anaesth 2001; 86: 859-68.

17. Frassanito L, Fiore V, Messina A, Bronzo V, Gilardi E, Vergari A. Effects of interscalene brachial plexus block on heart rate variability. Eur J Anaesthesiol 2011; 28(Suppl 48): 117.

18. Long TR, Wass CT, Burkle CM. Perioperative interscalene blockade: an overview of its history and current clinical use. J Clin Anesth 2002; 14: 546-56.

19. Seltzer JL. Hoarseness and Horner's syndrome after interscalene brachial plexus block. Anesth Analg 1977; 56: 585-6.

20. Vester-Andersen T, Christiansen C, Hansen A, Sorensen M, Meisler C. Interscalene brachial plexus block: area of analgesia, complications and blood concentrations of local anesthetics. Acta Anaesthesiol Scand 1981; 25: 81-4.

21. Sukhani R, Barclay J, Aasen M. Prolonged Horner's syndrome after interscalene block: a management dilemma. Anesth Analg 1994; 79: 601-3.

22. Guntamukkala M, Hardy PA. Spread of injectate after stellate ganglion block in man: an anatomical study. Br J Anaesth 1991; 66: 643-4.

23. Al-Khafaji JM, Ellias MA. Incidence of Horner syndrome with interscalene brachial plexus block and its importance in the management of head injury. Anesthesiology 1986; 64: 127.

24. Borgeat A, Ekatodramis G, Kalberer F, Benz C. Acute and nonacute complications associated with interscalene block and shoulder surgery. Anesthesiology 2001; 95: 875-80.

25. Rogers MC, Battit G, McPeek B, Todd D. Lateralization of sympathetic control of the human sinus node: EEG changes of stellate ganglion block. Anesthesiology 1978; 48: 139-41.

26. Kashima T, Tanaka H, Minagoe S, Toda H. Electrocardiographic changes induced by stellate ganglion block in normal subjects. J Electrocardiol 1981; 14: 169-74.

27. Saxena AK, Saxena N, Aggarwal B, Sethi AK. An unusual complication of sinus arrest following right-sided stellate ganglion block: a case report. Pain Practice 2004; 4: 245-8.

28. Koyama S, Sato N, Nagashima K, Aizawa H, Kawamura Y, Hasebe $\mathrm{N}$, et al. Effects of right stellate ganglion block on the autonomic nervous function of the heart: a study using the head-up tilt test. Circ J 2002; 66: 645-8.

29. Fujiki A, Masuda A, Inoue H. Effects of unilateral stellate ganglion block on the spectral characteristics of heart rate variability. Jpn Circ J 1999; 63: 854-8. 